\title{
Systems biology in inflammatory bowel diseases: on the way to precision medicine
}

\author{
Nikolas Dovrolis, Eirini Filidou, George Kolios \\ Democritus University of Thrace, Alexandroupolis, Greece
}

\begin{abstract}
Inflammatory bowel diseases (IBD) are chronic and recurrent inflammatory disorders of the gastrointestinal tract. The elucidation of their etiopathology requires complex and multiple approaches. Systems biology has come to fulfill this need in approaching the pathogenetic mechanisms of IBD and its etiopathology, in a comprehensive way, by combining data from different scientific sources. In combination with bioinformatics and network medicine, it uses principles from computer science, mathematics, physics, chemistry, biology, medicine and computational tools to achieve its purposes. Systems biology utilizes scientific sources that provide data from omics studies (e.g., genomics, transcriptomics, etc.) and clinical observations, whose combined analysis leads to network formation and ultimately to a more integrative image of disease etiopathogenesis. In this review, we analyze the current literature on the methods and the tools utilized by systems biology in order to cover an innovative and exciting field: IBD-omics.
\end{abstract}

Keywords Inflammatory bowel diseases, omics, IBD-omics, systems biology, bioinformatics

Ann Gastroenterol 2019; 32 (3): 1-14

\section{Introduction}

Complexity is one of the first words which come to mind when trying to decipher human health and the mechanisms behind the operation of cells, tissues and biological systems in general are vast and intertwined. Chronic and recurrent inflammatory disorders, such as inflammatory bowel diseases (IBD) and their 2 main representatives Crohn's disease (CD) and ulcerative colitis (UC), require complex and multiple approaches so that one can study every aspect of their etiopathology. Fig. 1 showcases the complex disease background of IBD, which in turn requires sophisticated approaches for its elucidation.

Systems biology [1], systems bioinformatics [2], computational biology [3], network medicine [4], and several other terms have been coined in recent years to denote the interdisciplinary field whose goal is to approach health as the integration of individual biological data and functions, using principles from computer science, mathematics, physics, chemistry, and computational tools. Omics data (genomics,

Laboratory of Pharmacology, Faculty of Medicine, Democritus University of Thrace, Alexandroupolis, Greece

Conflict of Interest: None

Correspondence to: Prof. George Kolios, MD PhD, Laboratory of Pharmacology, Faculty of Medicine, Democritus University of Thrace, Dragana, Alexandroupolis, 68100, Greece, e-mail: gkolios@med.duth.gr

Received 17 December 2018; accepted 25 February 2019; published online 3 April 2019

DOI: https://doi.org/10.20524/aog.2019.0373 transcriptomics, metabolomics, metagenomics, etc.; Table 1), along with clinical data and observations, are analyzed and associated with specific phenotypes forming networks of interacting entities. The suffix "-omics" in the name of a scientific field (e.g., genomics) refers to the comprehensive or global study of factors that may be implicated in disease pathogenesis [5].

In recent years, the principles and practices of systems biology have been applied to a multitude of human health aspects, allowing scientists to gain better insights and help drive therapeutic approaches. In unifactorial and multifactorial conditions alike, computational tools and algorithmic implementations have produced many critical observations in the study of obesity [6,7], diabetes [8,9], cardiovascular disease [10,11], autoimmune- [12,13] and inflammation-related conditions [14,15], neurodevelopment and neurodegeneration [16,17], lung disorders [18,19], cancer $[20,21]$, and a variety of other diseases. Computational drug discovery [22,23] and repositioning [24,25] have also benefited greatly from systemic approaches, allowing chemical compounds and molecules to be studied for targets in the mechanisms behind specific diseases.

In this review, we cover an innovative and exciting field: IBD-omics.

\section{IBD-omics}

IBD etiopathology is not completely known, yet various factors from different origins and systems are implicated in it [26]. Among these, it is known that the genetic background and the epigenetic modifications play an important role 
in disease predisposition, prognosis, and response to treatment [27]. It is also known that environmental factors may exacerbate or ameliorate patients' clinical symptoms and that the microbial composition and the metabolic profile of IBD patients differ from healthy individuals and may be the result or the cause of the disease's manifestation $[28,29]$. Additionally, there is accumulated knowledge on how the immune system and several expressed proinflammatory and profibrotic molecules are involved in disease pathogenesis [30]. However, these factors do not act alone: on the contrary, they synergize and shape the complex pathophysiology of IBD.

IBD-omics examine specific features involved in IBD, highlighting the imperative to employ methodologies that study and model all the existing interactions leading to knowledge acquisition (Fig. 2). In the subsequent sections, we identify and discuss the various molecular systems that shape the IBD field.

\section{Genomics in IBD}

DNA, the fundamental basis of life, was first discovered in 1869 by a Swiss physician and biologist, Johannes Friedrich

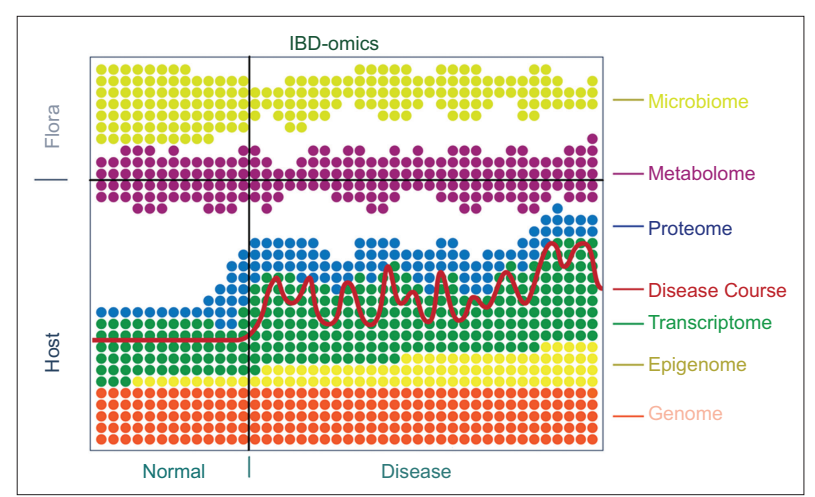

Figure 1 Graphical representation of -omes studied by inflammatory bowel disease (IBD)-omics: The genome (red) remains unchanged regardless of disease phase, whereas the epigenome (yellow) could be enriched as the disease progresses. During the disease the transcriptome (green) and proteome (blue) vary, usually increasing, while the gut microflora (dark-yellow) loses biodiversity. Finally, the metabolome (magenta) presents high variability, affected by both the host and the microflora
Miescher [31]. Since then, huge progress has been made, including the discovery of the DNA composition by Albrecht Kossel and Phoebus Levene and its structure by Watson and Crick; however, most important was the initiation and completion of the Human Genome Project (HGP), which succeeded in sequencing the whole human genome [32]. The term "genome" refers to the total gene composition of each organism and in humans, only about $2 \%$ of the genome ( $\sim 20,000$ genes) is translated into proteins. Nonetheless, the rest of the genome does not contain "junk" sequences, as first believed, but ones important for cell survival, functionality and evolution, such as non-coding RNAs, regulatory DNA regions, LINEs, SINEs, and introns [33].

Another goal of the HGP was to identify single nucleotide polymorphisms (SNPs), a single nucleotide substitution in DNA, which have been found to be the most common genetic variants and were later used in genome-association studies [34]. During the early 2000s, the first genome-wide association studies (GWAS) started to emerge and it was Klein et al that published the first GWAS on age-related macular degeneration, highlighting several gene mutations associated with this disease [35]. Since then, the number of articles reporting GWAS on various diseases has grown exponentially [36].

The basic principle of GWAS is that complex genetic diseases are associated with multiple and often common genetic polymorphisms; they are thus referred to as polygenic. The first part of GWAS methodology includes whole DNA extraction from patient and control samples, such as blood, followed by genetic sequencing for SNPs of specific genes, as described in the Methodology section of Guo et al [37]. For this purpose, chips containing genome-wide SNPs have been developed to test for common genetic variations among the population. Next, results are analyzed using various approaches. If an SNP frequently appears in the patient group, with a very high statistical significance, such as $\mathrm{P}<5 \times 10^{-8}$, then the genetic region that includes this $\mathrm{SNP}$ is considered to be a risk locus and therefore associated with the disease [38]. In other words, GWAS may accurately associate any specific SNP with a disease trait, outcome, or even response to treatment, excluding at the same time any insignificant difference between patients and controls $[39,40]$.

Even before the GWAS era, hypothesis-driven studies reported the first gene to be associated with susceptibility

Table 1 Definitions of omics

\begin{tabular}{ll}
\hline Type of omics & Definition \\
\hline $\begin{array}{l}\text { Genomics } \\
\text { Epigenomics }\end{array}$ & $\begin{array}{l}\text { Multidisciplinary approach to studying, quantifying and characterizing all the genes and their mutations in a given sample } \\
\text { The scientific field that studies the environment and genome interactions and the manner that these interactions may }\end{array}$ \\
\hline $\begin{array}{l}\text { Transcriptomics } \\
\text { The field of science that measures RNA expression in samples of various tissues and } \\
\text { allows for measurable differences and identification of how gene interactions transpire }\end{array}$ \\
$\begin{array}{ll}\text { Offers the opportunity for large-scale detection, identification and characterization of the whole protein expression of a } \\
\text { given cell or tissue }\end{array}$ \\
\hline Metabolomics & $\begin{array}{l}\text { The study of metabolic processes and changes in metabolite production in an organism } \\
\text { The total genetic composition of the microflora in a specific organ }\end{array}$ \\
\hline
\end{tabular}




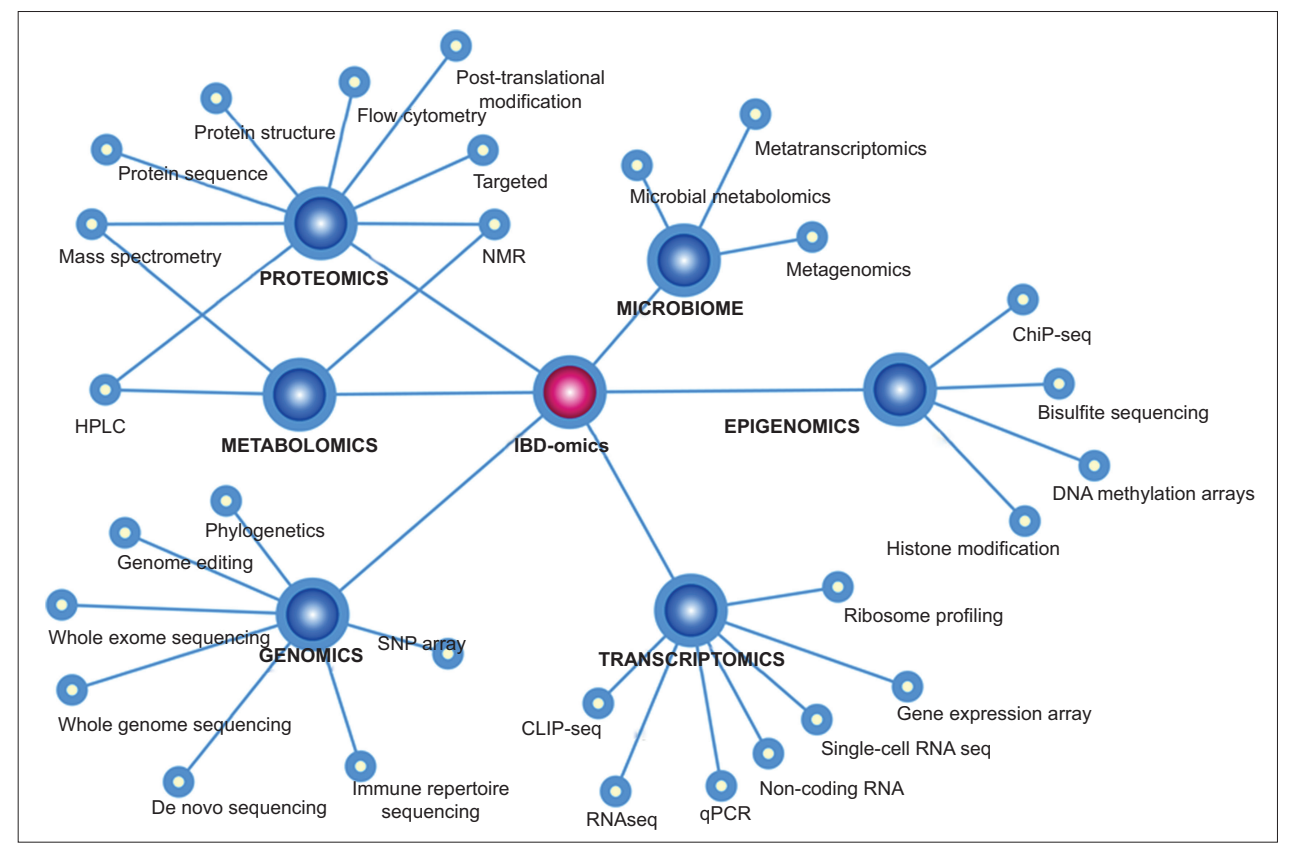

Figure 2 The plethora of methodologies and their experimental results, which can be combined to elucidate inflammatory bowel disease pathophysiology and molecular background

IBD-omics, inflammatory bowel disease-omics; NMR, nuclear magnetic resonance; HPLC, high-performance liquid chromatography; SNP, single nucleotide polymorphism; CLIP-seq, cross-linking immunoprecipitation-sequencing; RNAseq, RNA sequencing; qPCR, quantitative polymerase chain reaction; ChIP-seq, chromatin immunoprecipitation sequencing

to $\mathrm{CD}$, the nucleotide-binding oligomerization domaincontaining protein 2 (NOD2) [41,42]. NOD2 plays a significant role in inflammation as it is implicated in the activation of nuclear factor (NF)- $\kappa \mathrm{B}$, responsible for the activation of numerous genes of the innate and adaptive immunity [43]. In 2006, Duerr et al published the first GWAS on IBD and reported a strong association between $\mathrm{CD}$ patients and an SNP located in the IL23R gene [44]. Since then, many more GWA studies took place, highlighting susceptibility loci in IBD patients, such as ATG16L1, IRGM, TNFSF15, PTPN2, IL12B, $J A K 2$, STAT3 and many more that showed strong association with CD patients [38].

Another technological milestone in genomics was the development of the Immunochip. Based on the observation that many chronic and autoimmune diseases share common genetic traits, researchers developed a chip, the Immunochip, which contained about 200,000 SNPs and 800 small insertiondeletions found to be associated with different autoimmune disorders $[39,45]$. Using this technology, Jostins et al carried out a large scale meta-analysis and identified 163 genetic loci associated with CD, UC, or both [46]. Since then, the use of Immunochip has highlighted new genetic variants that are associated with IBD, the clinical course of the disease and even adverse events following treatment with anti-tumor necrosis factor (TNF) agents [47-49].

As mentioned above, GWA studies are limited in associating only common genetic variants with a disease trait, outcome or response to a treatment. This limitation was overcome by next-generation sequencing (NGS) technologies, which focus on studying rare genetic variants [40]. NGS not only offered the opportunity to study rare genetic variants, but also sped up the sequencing process and significantly lowered its cost. One of the technologies that emerged during the NGS era was targeted genome sequencing, where only parts of the genome are selectively sequenced and studied; this is accomplished by the use of DNA or RNA probes that specifically target and bind to the regions of interest in the DNA sequence. Whole exome sequencing (WES) is one example that arose from the use of these technologies [50].

WES aims at sequencing only the part of the genome that is translated into proteins [40]. Previous WES studies have identified new genetic variants associated with susceptibility to IBD, such as missense mutations in the genes PRDM1, NDP52, IL17REL, and CSF2RB [51-53]. A recent WES study on an Ashkenazi Jewish IBD population highlighted genetic variants in the genes NOD2, ZNF366 and MDGA1 associated with IBD, but failed to confirm previous associations with genes such as THEMIS, MCOLN2 and NLRP2 [54]. Similarly, Onoufriadis et al found a strong association between a rare variant in the NLRP7 gene and UC patients who originated from the UK, but not with CD patients, probably due to the small size of the CD patient group [55]. Very early onset IBD (VEOIBD), a severe variety of IBD that may manifest in children, has also been the focus of WES studies and researchers have identified rare genetic variants in several genes associated with VEOIBD, such as NOX1, NOD2, IL10RA and ADAM17 [56-60].

Genomic studies have shown that CD and UC may share the same genetic risk factors for susceptibility, but have also reported different genetic loci specifically associated with 
either disease or either disease's subphenotype. This discovery may offer clinicians a useful tool for distinguishing CD's subphenotypes and $\mathrm{CD}$ from UC, especially in cases where clinical and endoscopic criteria fail to do so [61]. Nonetheless, the genetic background of the population plays a significant role, as some genetic risk factors seem to be ethnic-specific, with different frequencies being reported in studies that include patients of different ethnicities [62-65].

\section{Epigenomics in IBD}

Beyond genetic factors implicated in $\mathrm{CD}$ and UC, which account for $13.6 \%$ and $7.5 \%$, of disease diversity respectively, environmental factors also contribute to IBD pathogenesis and may affect and alter the genetic background. Environmental elements that interact with DNA components and regulate its function are called epigenetic factors. Epigenetics is the field of science that studies the interactions between environment and genome and the manner that these interactions may regulate gene expression. DNA methylation and histone modifications are the main epigenetic modifications. Although they were first believed to be non-inheritable, recent studies suggest the opposite and thus may play a significant role in IBD pathogenesis [66].

DNA methylation occurs when the DNA methyltransferases (DNMTs) transfer a methyl group to cytosines, resulting in the formation of 5-methylcytosine. If this reaction takes place in regions of gene promoters, then it leads to obstruction of transcriptional factor binding and ultimately, to suppression of gene expression. DNA methylation is also implicated in histone modification, and together they regulate the expression patterns of cells $[67,68]$. The most common method for studying DNA methylation was first described by Frommer et al. Briefly, addition of sodium bisulfite to genomic DNA results in the conversion of non-methylated cytosines to uracils, while leaving methylated cytosines intact. Next, the methylation status of the gene of interest is calculated by performing methylation-specific polymerase chain reaction (PCR) [69].

In IBD, 3 types of DNMTs have been found to be implicated in its pathogenesis: DNMT1, DNMT3a and DNMT3b. DNMT1 and DNMT3b have been found to be elevated in inflamed mucosa sites of UC patients, while genetic variants of the DNMT3a gene have been associated with CD [70]. In a methylation-profiling study that included female-only CD patients, Li Yim et al found more than 4000 positions to be differentially methylated and to be associated with over 2700 genes in CD patients, out of which two were the most significant and located in PTPRN2 and BCL11A genes. The same investigators also identified 8 differentially methylated regions (DMRs) close to 8 different genes, and some of these genes were associated with immune-related pathways [71]. Regarding UC patients, Kang et al also found differentially methylated patterns in UC patients with 3 genes, FAM217B, KIAA1614 and RIBC2, being hypermethylated; thus, this might be a tool for distinguishing UC patients from healthy individuals [72]. In a recent study, mucosal biopsies taken from UC patients showed different methylation patterns from those of healthy individuals; UC patients showed hyper-methylation or hypo-methylation in genes involved in homeostasis and defense, or in immune response pathways, respectively [73]. An epigenome-wide association study revealed that IBD patients bear differentially methylated positions (DMPs) compared with healthy individuals and most of these DMPs found in IBD cases were shared between $\mathrm{CD}$ and UC patients. Among the top IBD-associated DMPs were positions located in the RPS6KA2, IL23A and TNFSF10 genes and among the IBD-related DMRs were regions near the genes VMP1, ITGB2, WDR8 and TXK [74]. In a similar study that included pediatric patients, Howell et al found that intestinal epithelial cells from IBD patients had different methylated patterns from controls and the methylation pattern was not only disease-specific but also gut-segment specific [75].

Histone modifications are another epigenetic mechanism that regulates gene expression; among these, histone acetylation and methylation are the best studied [76]. Histone acetylation takes place when a histone acetyl transferase adds an acetyl group to the amino-acid lysine of the histones, resulting in transcriptional enabling, while its removal by histone deacetylases (HDACs) leads to transcription blocking. On the other hand, histone methylation can either enable or block transcription, depending on the region where the methyl group is attached [27,76]. A well-established method for studying histone modifications is the use of chromatin immunoprecipitation (ChIP) protocol, where the chromatin structure is extracted, fragmentated and immunoprecipitated and, finally, the DNA is studied using various protocols, such as microarrays (ChIP-on-chip) or next-generation sequencing (ChiP-seq), that will enable the detection and quantification of modifications that occurred at the point of interest [77-79].

There are only few data regarding histone modifications in IBD. Bai et al found that lysine acetyltransferase 2B expression was significantly reduced in inflamed tissues of IBD patients, which resulted in low levels of histone $\mathrm{H} 4$ lysine 5 acetylation and, subsequently, in downregulation of interleukin (IL)-10 expression [80]. Previous in vitro studies have shown that Th17 immunological responses may be subject to histone modifications. Primary Th17 cells isolated from healthy individuals expressed high levels of IL-17A, and not IL-17F, upon stimulation with prostaglandin E2 and/or IL-23 plus IL-1 $\beta$. Further investigation revealed that the expression of 2 cytokines, IL-17A and IL-17F, was regulated by histone modifications; IL-17F expression was silenced due to histone methylation of $\mathrm{H} 3$, whereas IL-17A was overexpressed as a result of different patterns of methylation and acetylation of H3 [81]. Along similar lines, Ghadimi et al showed that certain commensal probiotics may inhibit the NF- $\mathrm{kB}$ transcriptional factor by reducing the histone acetylation levels [82]. Finally, data from murine models of colitis suggest that the inhibition HDACs may lead to the induction of apoptosis and Foxp3 expression and to the suppression of proinflammatory cytokine expression [76]. 


\section{Transcriptomics in IBD}

If we consider the genome to be the answer to "who is responsible for our genetic and health background?", the transcriptome answers the question "what does the genome do at any given time/cell?". As DNA gets transcribed to RNA, by RNA polymerase in the nucleus [83], it allows for measurable differences and identification of how gene interactions transpire (infer function via gene expression [84]) and what genetic information gets passed along in the "genotype to phenotype" pipeline. We now know that a considerable number of genes are to be transcribed to RNA and translated into proteins, while others regulate and assist as a variety of RNAs. Messenger RNA (mRNA) is our main source of characterizing coding genes while ribosomal (rRNA), micro (miRNA), long noncoding (lncRNA), transfer (tRNA), small nuclear (snRNA) and others are performing functions that make protein synthesis possible [85-87].

One of the advantages of modern technological advances has been the ability to measure gene expression in samples of various tissues after RNA isolation [88,89]. Quantitative real-time PCR (qPCR) [90], cap analysis gene expression (CAGE) [91], serial analysis of gene expression (SAGE) [92], microarrays [93], and total RNA sequencing (RNA-seq) [94] are just a few of the methodologies allowing us to quantify RNA transcripts. Differential gene expression analysis (DGEA) [95-97] enables studying the expression of specific genes under various conditions, tissues and timepoints and the juxtaposition between them to detect statistically significant differences that may signify association. For example, if a gene is found to be over- or under-expressed in several diseaseassociated samples versus controls, it allows for the assumption that this gene is implicated in the pathophysiology of the disease and the signaling pathways it is involved in are affected. Databases like KEGG [98] and REACTOME [99], supported by bioinformatics platforms like Enrichr [100] and Ingenuity Pathway Analysis (IPA) [101], enable researchers to identify these pathways and perform further analyses based on them.

In the past decades these techniques, along with various other omics, have assisted in identifying the pathophysiological mechanisms of IBD. In 2005 Costello et al [102], using cDNA arrays of colonic mucosa samples, identified several genes associated with UC and CD, highlighting the complex nature of IBD but also distinguishing the phenotypes. Following a similar motif, Schmidt et al [103] observed that IL-23p19 and IL-27p28 are elevated in CD but not in UC. Carey et al [104] highlighted IL-6:STAT3-dependent biological networks upregulated in IBD patients, regulating leukocyte recruitment, HLA expression, angiogenesis and tissue remodeling. Bamias et al [105] studied the difference in mucosal expression of housekeeping genes during IBD and concluded that it is altered, proposing other genes (namely RPLPO and RPS9) as reference and extended validation. Sugihara et al [106] described elevated C3 and IL-17 mRNA expressions in the inflamed mucosa of IBD patients. In Fransen et al [107], a correlation between IBD and IL6, IL23A and RORC was identified. Chiriac et al [108], using RNA-seq, found that colon tissues from IBD patients and mice with DSS colitis exhibited increased expression of IL28 versus controls, leading them to test and validate that IL28 administration in the animal model promotes mucosal healing. Hong et al [109], via RNA-seq, pinpointed differences between the inflamed and non-inflamed intestinal mucosa of CD patients and healthy controls. Of high interest in recent years is the study of noncoding RNAs and their role, as depicted in reviews and original articles [110-116].

Finally, Telesco et al [117], Arijs et al [118], Nunes et al [119], Lucafò et al [120], and Váradi et al [121], among others, have studied the response to specific therapeutic interventions to identify molecular targets and separate responders from nonresponders.

\section{Proteomics in IBD}

The term "proteome" refers to the total proteins, including all isoforms or post-translational modifications that can be expressed by the genome. Thus, proteomic analysis provides an opportunity for the large scale detection, identification and characterization of the whole protein expression of a given cell or tissue, making it the ideal tool for biomarker discovery [122]. Currently, there are several different approaches to proteomic analysis; in this review we try to briefly cover most of them.

Liquid chromatography and mass-spectrometry (MS) are the 2 widely used techniques for protein separation and identification. Liquid chromatography aims at separating the components found in a mixture, depending on their size, shape, charge or affinity for a certain ligand; there are thus many different types of chromatography [123]. MS is used for the identification of proteins, peptides or their post-translational modifications, and over the years several different techniques have been developed, from electrospray ionization (ESI) and matrix-assisted laser desorption/ionization (MALDI) to the new generation of mass analyzers and complex multistage instruments, such as hybrid quadrupole time-of-flight (Q-QTOF) [124]. Nonetheless, the most common MS techniques include surface-enhanced laser desorption/ionization timeof-flight (SELDI-TOF) and MALDI time-time-of-flight (MALDI-TOF). Identification of proteins based on these techniques requires, first, protein digestion into peptides, then measurement of the mass-to-charge ratio in an electric field and, finally, investigation of the peptide mass signatures in relation to a database of known proteins [122]. Beyond liquid chromatography and MS, more complex and quantitative techniques have been developed, such as 2D-PAGE, 2D-DIGE, ICAT, SILAC, proteolytic ${ }^{18} \mathrm{O}$ labeling, iTRAQ and protein/ antibody arrays (chips); a technical description of these is beyond the scope of this review but they were nicely analyzed by Alex et al [122].

By using any of the aforementioned techniques, proteomics are able to compare expression profiles between IBD patients and controls, which may ultimately lead to biomarker discovery. Over 10 years ago, Meuwis et al performed the first proteomic analysis in serum samples of IBD patients and controls and found 4 possible biomarkers (PF4, MRP8, 
FIBA and Hpalpha2) that could discriminate IBD patients from healthy individuals, thus providing a possible diagnostic tool [125]. During the same year, a similar proteomic study on intestinal epithelial cells from IBD patients revealed expression differences between inflamed and non-inflamed colonic regions in molecular pathways that regulate signal transduction, stress response and energy metabolism; among these, the most outstanding differences were in the expression of programmed cell death protein 8 and annexin 2A [126]. Along similar lines, Nanni et al observed statistically significant expression changes in the intestinal epithelial cells of CD patients and healthy individuals; some of these proteins, such as heat shock protein 70 and tryptase alpha-1 precursor, were upregulated in CD patients, while others, such as the nuclear protein Annexin A1 were downregulated [127]. Another interesting study on mononuclear cells from IBD patients and healthy donors was able not only to distinguish the expression profiles of patients and controls, but also to discriminate between CD and UC patients [128]. In a recent proteomic analysis, Manfredi et al showed that proteins related to the acute phase response and the complement activity were upregulated in serum samples from IBD patients, whereas proteins implicated in protease function, blood coagulation, oxygen transport and lipoprotein metabolism were downregulated [129]. Expression differences in genes related to immune responses between IBD patients and controls have also been identified, and according to the disease location or behavior, these expression patterns may vary among CD subtypes [130]. Proteomic analysis of colonic mucosal-luminal interface aspirates from pediatric patients with IBD-associated colitis led to the identification of possible markers for the diagnosis of UC [131]. Apart from diagnostic biomarkers that can enable easier discrimination between IBD adult or pediatric patients and healthy individuals or CD and UC patients, proteomic studies have also revealed possible biomarkers associated with response to treatment. Specifically, Meuwis et al conducted a small pilot study in CD patients treated with infliximab and found that PF4 was associated with response to treatment [132].

\section{Metabolomics in IBD}

Metabolomics is the scientific field that studies the metabolic processes and changes in metabolite production that occur in an organism. Metabolomic studies are usually performed in noninvasive samples, such as blood, urine, stool and swabs, and the resulting metabolic profile is the outcome of at least 3 different sources; dietary compounds, xenobiotics from the environment, and metabolic products of the microflora. Therefore, metabolomics studies in IBD usually examine the metabolic relationship between the host and the gut microflora and the factors that may influence this relationship [133].

The 2 most common technologies used in the field of metabolomics are MS, analyzed in the previous section (Proteomics), and nuclear magnetic resonance (NMR)spectroscopy [133]. NMR is a widely used technique in chemistry that provides information about the molecular structure of the examined compounds and their absolute or relative concentration in the sample. The main differences between MS and NMR-spectroscopy concern the sensitivity, and the sample size and preparation required. MS is the more sensitive method and is able to detect and identify metabolites with a thousand times higher sensitivity than NMRspectroscopy. Regarding the sample size, MS methods usually require volumes of 10-30 $\mu \mathrm{L}$, whereas NMR samples need to be about $300 \mu \mathrm{L}$. Lastly, NMR methods do not require any specific sample preparation other than sample dilutions, whereas, as described above, MS requires a series of steps before sample analysis [134].

The first metabolomics study was performed in fecal samples from twin CD patients and twin healthy individuals and one of the findings was that the metabolic profile differed among patients with ileal disease, patients with colonic disease and healthy individuals. The differences in metabolite content were identified to be on pathways concerning the metabolism or synthesis of amino acids, fatty acids, bile acids and arachidonic acid; thus, this study suggested possible metabolic biomarkers for disease diagnosis and phenotype characterization [135]. Along similar lines, Bjerrum et al performed a metabolomic study in patients with active or inactive UC and healthy individuals and found that the metabolomic profile of colonic biopsies and colonic epithelial cells differed between active UC and controls. The most interesting result, however, was the fact that, although inactive UC patients were free of clinical and histological findings, the metabolic profile of $20 \%$ of them matched with that of active UC patients, suggesting again that metabolomic analysis might be a useful tool for disease prognosis [136]. In a recent study, Diab et al investigated the metabolite levels of omega- 3 and omega- 6 polyunsaturated fatty acids in colonic biopsies taken from healthy controls and treatment-naïve or deep remission UC patients. They found that levels of omega- 6 metabolites such as prostaglandin E2, thromboxane, trans-leukotriene, and 12-hydroxy-eicosatetraenoic acid, known to be actively involved in inflammation, were significantly higher in treatment-naïve UC patients compared with the other 2 groups, while levels of omega-3 metabolites were lower. Furthermore, the elevated levels of omega- 6 metabolites correlated with increased proinflammatory cytokine expression, suggesting that polyunsaturated fatty acid metabolism plays a significant role during the onset of the disease [137]. In another study, Keshteli et al highlighted metabolic differences in urine and serum samples between UC patients in remission or relapse. They showed that significantly higher levels of trans-aconitate, 3-hydroxybutyrate, acetoacetate and acetone, and lower levels of acetamide and cystine were found in UC patients experiencing a clinical relapse, suggesting possible biomarkers for disease prognosis [138]. Metabolomic signatures could also contribute to disease categorization, as in a recent study serum metabolic profiles differed among healthy individuals and UC or CD patients [139]. Apart from the host, changes in the metabolic profile of gut microflora have also been found between healthy individuals and IBD patients. One of the first studies that compared the metabolomic signatures in fecal samples from healthy individuals and patients with UC or 
irritable bowel syndrome showed that taurine and cadaverine levels were higher in UC patients [140]. Furthermore, our research group, using in silico approaches, has recently reported that the microbial metabolites of CD B2 or B3 behavioral subphenotypes differ from the B1 sub-phenotype, suggesting that metabolomic analysis might contribute to disease subphenotype classification [141].

Overall, metabolomics is a promising tool for disease diagnosis, prognosis and classification and its now low-cost and easy-to-perform evaluations could add significant information in everyday clinical practice.

\section{Microbiome in IBD: the meta-paradigm}

So far, this review has presented various -omics approaches that help characterize and analyze the background of human health. But is this the full picture or is it all a matter of application? The truth is that the targets of these techniques are usually only one tenth of the cells in the human body. What has happened in recent years is a paradigm shift towards exploring the rest; the identity and function of our symbionts. The microflora or microbiota, as they are referred to in the literature, are viruses, fungi, archaea, and, most importantly, bacteria that live primarily in the gastrointestinal tract but also on the skin, in the mouth, nose and lungs. Their total genetic composition is called the microbiome and has become the new focus for genomics, transcriptomics, metabolomics and various other targeted and blanket approaches using state-ofthe-art and established technologies [142-144].

Microbiota are usually satisfied staying in an ever-evolving cycle of mutualistic bliss (albeit a fragile one); the homeostasis. During homeostasis the microbiome's function [145] is associated with human health by providing (in an interaction with the gut mucosa and the immune system [146-148]) defense against pathogens, modulation of inflammation, production of energy and vitamins, and assistance with the host's metabolism and nutrient intake. When homeostasis becomes unbalanced and microbial populations and functions are altered, the phenomenon is called dysbiosis. It has been directly associated [149-151] with the onset, progression and therapeutic response of multiple health conditions, including lung-associated disorders [152,153], obesity [154,155], diabetes [156,157], cardiovascular disease and atherosclerosis [158-161], chronic kidney disease [162-164], cancer [165-167], neurological and neuropsychiatric disorders [168-171], and, in the spirit of this review, IBD [172-174].

As the microbiome comes under extensive scrutiny, new insights into its association with IBD have come to light to present a parallel alteration in behavior and phenotype [175]. In 2008 Huttenhower et al [176] identified Faecalibacterium prausnitzii's anti-inflammatory role in $\mathrm{CD}$, and later studies [177-179] confirmed its reduction during dysbiosis. Robinson et al [180], using an animal model of colitis, identified several phylogenetic and metabolic associated changes in the microbiome similar to what happens in human IBD. Chu et al [181] described how outer membrane vesicles secreted by
Bacteroides fragilis play a role in the immunomodulation of IBD in partnership with the NOD2 and ATG16L1 genes. Halfvarson et al [182], in a longitudinal study of the microbiome, proposed and demonstrated diversion from a bacterial healthy plane during IBD, while also studying the role of f-calprotectin without finding any statistically significant association. In a recent work, our group has also identified dysbiosis associated with the complex behavioral sub-phenotypes of CD (stricturing and penetrating) and the differential diversity and function of the microbiome versus the inflammatory sub-phenotype [183]. Ananthakrishnan et al [184] have studied the response to antiintegrin biologic therapy in association with the microbiome and have concluded that microbial function and diversity in early stages of therapy might be able to predict its efficacy. Finally, many studies have focused on fecal microbiota transplantation (FMT) [185-189] as a potential therapeutic action versus IBD, but also as a possible irritant [190,191].

\section{From systems biology to treating IBD}

As it stands, we now possess an abundance of tools and technical methodologies to analyze and try to comprehend the genetic and molecular background of IBD. This is, though, just the first step in the war waged against it. We are facing a group of diseases that differ greatly in their symptomology and progression, but also in the approaches that should be taken to treat them. Up to now, both CD and UC have been considered manageable but untreatable, with a heavy burden on the quality of life. This, combined with the long and tedious process of finding a potential therapeutic target, identifying/ validating a chemical compound/molecule and ultimately providing it to the general public, means that changing IBD's status to treatable may still be far away.

Systems biology has provided the tools to ameliorate the situation in all steps along the way. We have already discussed how -omics approaches highlight potential pharmacological targets, but what about drug identification and validation? Or their deployment and efficacy in the general population? Drug repositioning [192-194] has been introduced to costeffectively, accurately and efficiently identify drugs that can help regulate the pathology of a disease. It allows for repurposing pharmaceuticals already on the market to be used on new targets. These drugs have already undergone extensive preclinical and clinical trials, have known side-effects and are generally considered safe for the patient under other conditions. Computational drug repositioning, because of its nature in utilizing -omics data, is a step towards precision medicine. By identifying drugs for specific patients/patient groups, there is no need to classify and account for responders and non-responders to treatment, since the drugs discovered are specifically identified using the patient's genetic and molecular background [195-199].

Over the years, by using all the aforementioned approaches of -omics, possible therapeutic targets have been identified, and this has led to the development of biological therapies and, ultimately, to personalized medicine. The milestone during 
Table 2 Inflammatory bowel disease (IBD)-omics, the expected outcome, and its possible applications in clinical practice

\begin{tabular}{|c|c|c|}
\hline IBD-omics & Expected outcome & Possible applications \\
\hline Genomics & DNA sequencing, SNP identification & \multirow{6}{*}{$\begin{array}{l}\text { Predisposition, } \\
\text { diagnosis, } \\
\text { prognosis, } \\
\text { staging, } \\
\text { disease sub-phenotype classification, } \\
\text { response to treatment }\end{array}$} \\
\hline Epigenomics & Differential patterns of DNA and histone modifications & \\
\hline Transcriptomics & $\begin{array}{l}\text { Differential expression patterns of RNA (mRNA, rRNA, } \\
\text { miRNA, IncRNA, tRNA, snRNA) }\end{array}$ & \\
\hline Proteomics & $\begin{array}{l}\text { Detection, identification and characterization of whole } \\
\text { protein expression levels }\end{array}$ & \\
\hline Metabolomics & Detection and identification of metabolite composition & \\
\hline Microbiome & $\begin{array}{l}\text { Detection, identification and characterization of } \\
\text { microbial composition and its metabolic profile }\end{array}$ & \\
\hline
\end{tabular}

SNP, single nucleotide polymorphisms

all those years of research was the use of anti-TNF agents in treating IBD patients. Infliximab was the first chimeric monoclonal antibody against TNF- $\alpha$, a proinflammatory cytokine with a central role in systemic inflammation. Since then, 3 other anti-TNF agents have been approved for IBD; adalimumab, certolizumab pegol, and golimumab [200]. Beyond anti-TNF agents, many other biological therapies against proinflammatory ILs, such as ustekinumab, intracellular signaling targets, such as Janus kinase inhibitors (tofacitinib, filgotinib, and upadacitinib), and cell adhesion molecules, such as natalizumab, vedolizumab, etrolizumab, AJM300 and PF-00547659 either have been approved for IBD treatment or are currently under investigation in clinical trials [200-204].

\section{Biological data integration and networks: the way forward}

As with all multidisciplinary approaches, medical systems biology must advance on multiple fronts to be effective. Its goals and hypotheses must be reassessed and its methodologies must be updated constantly. For example, multidisciplinary approaches such as the recently emerged "proteogenomics" (a combination of proteomics, genomics, and transcriptomics) can allow for a holistic point of view regarding disease pathophysiology [205]. Medicine has moved away from a generalized treatment-oriented goal to preventive and personalized approaches. The target is no longer just to treat a condition, but how to do it effectively and efficiently while gaining a better understanding of its methods of action. Meanwhile, biological data acquisition and digitization techniques (omics) advance every day, producing higher volumes of data with higher specificity and precision. Computational power and toolkits must keep up with these new challenges. New databases, algorithms and user-friendly applications [206211] are being developed to support the analysis of said data by experts in bioinformatics and physicians alike.

The application of graph and network theory principles in the construction and analysis of biological networks is arguably the strongest weapon in our arsenal [212]. It provides the means to study multiple biological interactions in a mathematical way and extract information that might not be easily comprehensible otherwise [213]. Genes, proteins, microbiota, diseases, drugs and a variety of other entities can be linked together and analyzed in a meaningful manner to extract significance and association. Perhaps the easiest way to understand biological networks is to think of a molecular signaling pathway and how genes synergize together to perform a function as a whole. But that is only the tip of the iceberg in our case; having the accumulated knowledge of past years we know which genes are co-expressed in various experiments, to which proteins these are being translated to, how those interact amongst themselves, how they can provide variability in the course of a disease or its treatment, and so forth.

We can approach these networks via different methodologies and tools depending on what our target is. Probably the most commonly used paradigms include GENEMANIA [214] for gene interactions, STRING [215] for protein-protein associations, KEGG [216] or REACTOME [99] for signaling pathways, and even some powerful solutions like Cytoscape [217] or the IGRAPH [218] package for R oriented towards more tech-savvy users, but the whole list is very extensive.

In IBD, network approaches are not very popular, with less than 400 papers utilizing some kind of network implementation having been indexed in PubMed over the last 3 years. Some recent examples of different kinds of networks include the work of Peters et al [219], with the creation of a complex genomic network via a variety of resources and its modeling and analysis, the work of Benchimol et al [220], who employed distributed network analysis on phenotypic and locational data to identify the epidemiology of IBD in Canada, and how Coward et al [221] used a network based meta-analysis to compare the effectiveness of commonly used IBD treatments.

\section{Concluding remarks}

The sections of this review have covered the most popular -omics, how they are applied and their implementation in IBD studies. Table 2 showcases the results we can expect from -omics analyses along with how these can be interpreted and utilized in everyday clinical practice. This is important because, regardless of the methodology, the ultimate goal will always be the usability and effectiveness of the results. We neither want nor need more meaningless data, but rather 
clear information about how to approach, evaluate and treat a disorder. It is apparent that systems biology is years away from being the only solution we will ever need to employ again, but is nevertheless a very efficient way to elucidate the knowledge we currently have and continue to amass.

\section{References}

1. Ideker T, Galitski T, Hood L. A new approach to decoding life: systems biology. Annu Rev Genomics Hum Genet 2001;2:343-372.

2. Oulas A, Minadakis $G$, Zachariou $M$, et al. Systems bioinformatics: increasing precision of computational diagnostics and therapeutics through network-based approaches. Brief Bioinform 2017 Nov 27 [Epub ahead of print]. doi: 10.1093/bib/bbx151.

3. Waterman MS, Introduction to computational biology: maps, sequences and genomes. CRC Press: Boca Raton, FL, USA; 1995.

4. Barabási AL, Gulbahce N, Loscalzo J. Network medicine: a network-based approach to human disease. Nat Rev Genet 2011; 12:56-68.

5. Hasin Y, Seldin M, Lusis A. Multi-omics approaches to disease. Genome Biol 2017;18:83.

6. Locke AE, Kahali B, Berndt SI, et al; International Endogene Consortium. Genetic studies of body mass index yield new insights for obesity biology. Nature 2015;518:197-206.

7. Levian C, Ruiz E, Yang X. The pathogenesis of obesity from a genomic and systems biology perspective. Yale J Biol Med 2014; 87:113-126.

8. Eizirik DL, Moore F, Flamez D, Ortis F. Use of a systems biology approach to understand pancreatic beta-cell death in Type 1 diabetes. Biochem Soc Trans 2008;36:321-327.

9. Wang-Sattler R, Yu Z, Herder C, et al. Novel biomarkers for prediabetes identified by metabolomics. Mol Syst Biol 2012;8:615.

10. Meng Q, Mäkinen VP, Luk H, Yang X. Systems biology approaches and applications in obesity, diabetes, and cardiovascular diseases. Curr Cardiovasc Risk Rep 2013;7:73-83.

11. Gray WD, French KM, Ghosh-Choudhary S, et al. Identification of therapeuticcovariantmicroRNAclustersinhypoxia-treatedcardiac progenitor cell exosomes using systems biology. Circ Res 2015; 116:255-263

12. Lorenzon R, Mariotti-Ferrandiz E, Aheng C, et al. Clinical and multi-omics cross-phenotyping of patients with autoimmune and autoinflammatory diseases: the observational TRANSIMMUNOM protocol. BMJ Open 2018;8:e21037.

13. Tsang JS. Utilizing population variation, vaccination, and systems biology to study human immunology. Trends Immunol 2015;36: 479-493.

14. Hotamisligil GS. Inflammation, metaflammation and immunometabolic disorders. Nature 2017;542:177-185.

15. Starchenko A, Lauffenburger DA. In vivo systems biology approaches to chronic immune/inflammatory pathophysiology. Curr Opin Biotechnol 2018;52:9-16.

16. Parikshak NN, Gandal MJ, Geschwind DH. Systems biology and gene networks in neurodevelopmental and neurodegenerative disorders. Nat Rev Genet 2015;16:441-458.

17. Talwar P, Gupta R, Kushwaha S, et al. Viral induced oxidative and inflammatory response in Alzheimer's disease pathogenesis with identification of potential drug candidates: A systematic review using systems biology approach. Curr Neuropharmacol 2018 Apr 19 [Epub ahead of print]. doi: 10.2174/1570159X16666 180419124508.

18. Harvey LD, Chan SY. Evolving systems biology approaches to understanding non-coding RNAs in pulmonary hypertension.
J Physiol 2019;597:1199-1208.

19. Ferreira MA, Vonk JM, Baurecht $\mathrm{H}$, et al; LifeLines Cohort Study. Shared genetic origin of asthma, hay fever and eczema elucidates allergic disease biology. Nat Genet 2017;49:1752-1757.

20. Zielinski DC, Jamshidi N, Corbett AJ, Bordbar A, Thomas A, Palsson BO. Systems biology analysis of drivers underlying hallmarks of cancer cell metabolism. Sci Rep 2017;7:41241.

21. Agrawal P, Fontanals-Cirera B, Sokolova E, et al. A systems biology approach identifies FUT8 as a driver of melanoma metastasis. Cancer Cell 2017;31:804-819.

22. Moffat JG, Vincent F, Lee JA, Eder J, Prunotto M. Opportunities and challenges in phenotypic drug discovery: an industry perspective. Nat Rev Drug Discov 2017;16:531-543.

23. Ryall KA, Tan AC. Systems biology approaches for advancing the discovery of effective drug combinations. J Cheminform 2015;7:7.

24. Dovrolis N, Kolios G, Spyrou G, Maroulakou I. Laying in silico pipelines for drug repositioning: a paradigm in ensemble analysis for neurodegenerative diseases. Drug Discov Today 2017;22: 805-813.

25. Rastegar-Mojarad M, Ye Z, Kolesar JM, Hebbring SJ, Lin SM. Opportunities for drug repositioning from phenome-wide association studies. Nat Biotechnol 2015;33:342-345.

26. Valatas V, Filidou E, Drygiannakis I, Kolios G. Stromal and immune cells in gut fibrosis: the myofibroblast and the scarface. Ann Gastroenterol 2017;30:393-404.

27. Wawrzyniak M, Scharl M. Genetics and epigenetics of inflammatory bowel disease. Swiss Med Wkly 2018;148:w14671.

28. Levy M, Blacher E, Elinav E. Microbiome, metabolites and host immunity. Curr Opin Microbiol 2017;35:8-15.

29. Rapozo DC, Bernardazzi C, de Souza HS. Diet and microbiota in inflammatory bowel disease: The gut in disharmony. World $J$ Gastroenterol 2017;23:2124-2140.

30. Uhlig HH, Powrie F. Translating immunology into therapeutic concepts for inflammatory bowel disease. Annu Rev Immunol 2018; 36:755-781.

31. Dahm R. Friedrich Miescher and the discovery of DNA. Dev Biol 2005;278:274-288.

32. Moraes F, Góes A. A decade of human genome project conclusion: scientific diffusion about our genome knowledge. Biochem Mol Biol Educ 2016;44:215-223.

33. Lander ES, Linton LM, Birren B, et al; International Human Genome Sequencing Consortium. Initial sequencing and analysis of the human genome. Nature 2001;409:860-921.

34. Cargill M, Daley GQ. Mining for SNPs: putting the common variants-common disease hypothesis to the test. Pharmacogenomics 2000;1:27-37.

35. Klein RJ, Zeiss C, Chew EY, et al. Complement factor $\mathrm{H}$ polymorphism in age-related macular degeneration. Science 2005; 308:385-389.

36. Wang QL, Tan WL, Zhao YJ, et al. Data analysis in the postgenome-wide association study era. Chronic Dis Transl Med 2016;2:231-234.

37. Guo CC, Wei N, Liang SH, Wang BL, Sha SM, Wu KC. Populationspecific genome-wide mapping of expression quantitative trait loci in the colon of Han Chinese. J Dig Dis 2016;17:600-609.

38. Uniken Venema WT, Voskuil MD, Dijkstra G, Weersma RK, Festen EA. The genetic background of inflammatory bowel disease: from correlation to causality. J Pathol 2017;241:146-158.

39. McGovern DP, Kugathasan S, Cho JH. Genetics of inflammatory bowel diseases. Gastroenterology 2015;149:1163-1176.

40. Uhlig HH, Muise AM. Clinical genomics in inflammatory bowel disease. Trends Genet 2017;33:629-641.

41. Hugot JP, Chamaillard M, Zouali H, et al. Association of NOD2 leucine-rich repeat variants with susceptibility to Crohn's disease. Nature 2001;411:599-603.

42. Ogura $\mathrm{Y}$, Bonen DK, Inohara N, et al. A frameshift mutation in 
NOD2 associated with susceptibility to Crohn's disease. Nature 2001;411:603-606

43. McDaniel DK, Eden K, Ringel VM, Allen IC. Emerging roles for Noncanonical NF- $\kappa B$ signaling in the modulation of inflammatory bowel disease pathobiology. Inflamm Bowel Dis 2016;22:2265-2279.

44. Duerr RH, Taylor KD, Brant SR, et al. A genome-wide association study identifies IL23R as an inflammatory bowel disease gene. Science 2006;314:1461-1463.

45. Palmieri O, Mazza T, Castellana S, et al. Inflammatory bowel disease meets systems biology: a multi-omics challenge and frontier. OMICS 2016;20:692-698.

46. Jostins L, Ripke S, Weersma RK, et al; International IBD genetics consortium (IIBDGC). Host-microbe interactions have shaped the genetic architecture of inflammatory bowel disease. Nature 2012;491:119-124.

47. Borren NZ, Conway G, Garber JJ, et al. Differences in clinical course, genetics, and the microbiome between familial and sporadic inflammatory bowel diseases. J Crohns Colitis 2018;12: 525-531.

48. Hong $\mathrm{M}$, Ye $\mathrm{BD}$, Yang SK, et al. Immunochip meta-analysis of inflammatory bowel disease identifies three novel loci and four novel associations in previously reported loci. J Crohns Colitis 2018;12:730-741.

49. Lew D, Yoon SM, Yan X, et al. Genetic associations with adverse events from anti-tumor necrosis factor therapy in inflammatory bowel disease patients. World J Gastroenterol 2017;23:7265-7273.

50. Petersen BS, Fredrich B, Hoeppner MP, Ellinghaus D, Franke A. Opportunities and challenges of whole-genome and -exome sequencing. BMC Genet 2017;18:14.

51. Chuang LS, Villaverde N, Hui KY, et al. A frameshift in CSF2RB predominant among ashkenazi jews increases risk for Crohn's disease and reduces monocyte signaling via GM-CSF. Gastroenterology 2016;151:710-723.

52. Ellinghaus $\mathrm{D}$, Zhang $\mathrm{H}$, Zeissig $\mathrm{S}$, et al. Association between variants of PRDM1 and NDP52 and Crohn's disease, based on exome sequencing and functional studies. Gastroenterology 2013;145:339-347.

53. Sasaki MM, Skol AD, Hungate EA, et al. Whole-exome sequence analysis implicates rare Il17REL variants in familial and sporadic inflammatory bowel disease. Inflamm Bowel Dis 2016;22:20-27.

54. Schiff ER, Frampton M, Ben-Yosef N, et al. Rare coding variant analysis in a large cohort of Ashkenazi Jewish families with inflammatory bowel disease. Hum Genet 2018;137:723-734.

55. Onoufriadis A, Stone K, Katsiamides A, et al. Exome sequencing and genotyping identify a rare variant in NLRP7 gene associated with ulcerative colitis. J Crohns Colitis 2018;12:321-326.

56. Girardelli M, Loganes C, Pin A, et al. Novel NOD2 mutation in early-onset inflammatory bowel phenotype. Inflamm Bowel Dis 2018;24:1204-1212.

57. Shaw KA, Cutler DJ, Okou D, et al. Genetic variants and pathways implicated in a pediatric inflammatory bowel disease cohort. Genes Immun 2019;20:131-142.

58. Schwerd T, Bryant RV, Pandey S, et al. NOX1 loss-of-function genetic variants in patients with inflammatory bowel disease. Mucosal Immunol 2018;11:562-574.

59. Blaydon DC, Biancheri P, Di WL, et al. Inflammatory skin and bowel disease linked to ADAM17 deletion. N Engl J Med 2011; 365:1502-1508.

60. Glocker EO, Kotlarz D, Boztug K, et al. Inflammatory bowel disease and mutations affecting the interleukin-10 receptor. N Engl J Med 2009;361:2033-2045.

61. Ye BD, McGovern DP. Genetic variation in IBD: progress, clues to pathogenesis and possible clinical utility. Expert Rev Clin Immunol 2016;12:1091-1107.
62. Brant SR, Okou DT, Simpson CL, et al. Genome-wide association study identifies african-specific susceptibility loci in african americans with inflammatory bowel disease. Gastroenterology 2017;152:206-217.

63. Fuyuno Y, Yamazaki K, Takahashi A, et al. Genetic characteristics of inflammatory bowel disease in a Japanese population. J Gastroenterol 2016;51:672-681.

64. Huang C, Haritunians T, Okou DT, et al. Characterization of genetic loci that affect susceptibility to inflammatory bowel diseases in African Americans. Gastroenterology 2015;149: $1575-1586$

65. Juyal G, Prasad P, Senapati S, et al. An investigation of genomewide studies reported susceptibility loci for ulcerative colitis shows limited replication in north Indians. PLoS One 2011;6:e16565.

66. Cleynen I, Vermeire S. The genetic architecture of inflammatory bowel disease: past, present and future. Curr Opin Gastroenterol 2015;31:456-463.

67. Jin $\mathrm{B}, \mathrm{Li} \mathrm{Y}$, Robertson KD. DNA Methylation: superior or subordinate in the epigenetic hierarchy? Genes Cancer 2011;2: 607-617.

68. Bhattacharjee R, Moriam S, Umer M, Nguyen NT, Shiddiky MJA. DNA methylation detection: recent developments in bisulfite free electrochemical and optical approaches. Analyst 2018;143: 4802-4818.

69. Frommer M, McDonald LE, Millar DS, et al. A genomic sequencing protocol that yields a positive display of 5-methylcytosine residues in individual DNA strands. Proc Natl Acad Sci U S A 1992;89:1827-1831.

70. Low D, Mizoguchi A, Mizoguchi E. DNA methylation in inflammatory bowel disease and beyond. World J Gastroenterol 2013;19:5238-5249.

71. Li Yim AYF, Duijvis NW, Zhao J, et al. Peripheral blood methylation profiling of female Crohn's disease patients. Clin Epigenetics 2016;8:65.

72. Kang K, Bae J-H, Han K, Kim ES, Kim TO, Yi JM. A genomewide methylation approach identifies a new hypermethylated gene panel in ulcerative colitis. Int J Mol Sci 2016;17.

73. Taman H, Fenton CG, Hensel IV, Anderssen E, Florholmen J, Paulssen RH. Genome-wide DNA methylation in treatmentnaïve ulcerative colitis. J Crohns Colitis 2018;12:1338-1347.

74. Ventham NT, Kennedy NA, Adams AT, et al; IBD CHARACTER consortium. Integrative epigenome-wide analysis demonstrates that DNA methylation may mediate genetic risk in inflammatory bowel disease. Nat Commun 2016;7:13507.

75. Howell KJ, Kraiczy J, Nayak KM, et al. DNA Methylation and transcription patterns in intestinal epithelial cells from pediatric patients with inflammatory bowel diseases differentiate disease subtypes and associate with outcome. Gastroenterology 2018;154:585-598.

76. Fogel O, Richard-Miceli C, Tost J. Epigenetic changes in chronic inflammatory diseases. Adv Protein Chem Struct Biol 2017; 106:139-189.

77. Gerstein MB, Kundaje A, Hariharan M, et al. Architecture of the human regulatory network derived from ENCODE data. Nature 2012;489:91-100

78. Mikkelsen TS, Ku M, Jaffe DB, et al. Genome-wide maps of chromatin state in pluripotent and lineage-committed cells. Nature 2007;448:553-560.

79. Barski A, Cuddapah S, Cui K, et al. High-resolution profiling of histone methylations in the human genome. Cell 2007;129: 823-837.

80. Bai AH, Wu WK, Xu L, et al. Dysregulated lysine acetyltransferase 2B promotes inflammatory bowel disease pathogenesis through transcriptional repression of interleukin-10. JCrohns Colitis 2016; 10:726-734. 
81. Adamik J, Henkel M, Ray A, Auron PE, Duerr R, Barrie A. The IL17A and IL17F loci have divergent histone modifications and are differentially regulated by prostaglandin E2 in Th17 cells. Cytokine 2013;64:404-412.

82. Ghadimi D, Helwig U, Schrezenmeir J, Heller KJ, de Vrese M. Epigenetic imprinting by commensal probiotics inhibits the IL-23/IL-17 axis in an in vitro model of the intestinal mucosal immune system. J Leukoc Biol 2012;92:895-911.

83. Dignam JD, Lebovitz RM, Roeder RG. Accurate transcription initiation by RNA polymerase II in a soluble extract from isolated mammalian nuclei. Nucleic Acids Res 1983;11:1475-1489.

84. DeRisi JL, Iyer VR, Brown PO. Exploring the metabolic and genetic control of gene expression on a genomic scale. Science 1997; 278:680-686.

85. Taft RJ, Pang KC, Mercer TR, Dinger M, Mattick JS. Non-coding RNAs: regulators of disease. J Pathol 2010;220:126-139.

86. Tuck AC, Tollervey D. RNA in pieces. Trends Genet 2011;27: 422-432.

87. Yoon J-H, Abdelmohsen K, Gorospe M. Functional interactions among microRNAs and long noncoding RNAs. in Semin Cell Dev Biol 2014. Elsevier.

88. Chomczynski P, Sacchi N. Single-step method of RNA isolation by acid guanidinium thiocyanate-phenol-chloroform extraction. Anal Biochem 1987;162:156-159.

89. Chirgwin JM, Przybyla AE, MacDonald RJ, Rutter WJ. Isolation of biologically active ribonucleic acid from sources enriched in ribonuclease. Biochemistry 1979;18:5294-5299.

90. Ramakers C, Ruijter JM, Deprez RH, Moorman AF. Assumptionfree analysis of quantitative real-time polymerase chain reaction (PCR) data. Neurosci Lett 2003;339:62-66.

91. Shiraki T, Kondo S, Katayama S, et al. Cap analysis gene expression for high-throughput analysis of transcriptional starting point and identification of promoter usage. Proc Natl Acad Sci U S A 2003; 100:15776-15781.

92. Velculescu VE, Zhang L, Vogelstein B, Kinzler KW. Serial analysis of gene expression. Science 1995;270:484-487.

93. Schulze A, Downward J. Navigating gene expression using microarrays-a technology review. Nat Cell Biol 2001; 3:E190-E195.

94. Wang Z, Gerstein M, Snyder M. RNA-Seq: a revolutionary tool for transcriptomics. Nat Rev Genet 2009;10:57-63.

95. Anders S, Huber W. Differential expression analysis for sequence count data. Genome Biol 2010;11:R106.

96. Trapnell C, Roberts A, Goff L, et al. Differential gene and transcript expression analysis of RNA-seq experiments with TopHat and Cufflinks. Nat Protoc 2012;7:562-578.

97. Robinson MD, McCarthy DJ, Smyth GK. edgeR: a Bioconductor package for differential expression analysis of digital gene expression data. Bioinformatics 2010;26:139-140.

98. Kanehisa M, Goto S. KEGG: kyoto encyclopedia of genes and genomes. Nucleic Acids Res 2000;28:27-30.

99. Joshi-Tope G, Gillespie M, Vastrik I, et al. Reactome: a knowledgebase of biological pathways. Nucleic Acids Res 2005; 33:D428-D432.

100. Chen EY, Tan CM, Kou Y, et al. Enrichr: interactive and collaborative HTML5 gene list enrichment analysis tool. BMC Bioinformatics 2013;14:128.

101. Krämer A, Green J, Pollard J Jr, Tugendreich S. Causal analysis approaches in Ingenuity Pathway Analysis. Bioinformatics 2014; 30:523-530.

102. Costello CM, Mah N, Häsler R, et al. Dissection of the inflammatory bowel disease transcriptome using genome-wide cDNA microarrays. PLoS Med 2005;2:e199.

103. Schmidt C, Giese T, Ludwig B, et al. Expression of interleukin12-related cytokine transcripts in inflammatory bowel disease: elevated interleukin-23p19 and interleukin-27p28 in Crohn's disease but not in ulcerative colitis. Inflamm Bowel Dis 2005; 11:16-23.

104. Carey R, Jurickova I, Ballard E, et al. Activation of an IL-6: STAT3dependent transcriptome in pediatric-onset inflammatory bowel disease. Inflamm Bowel Dis 2008;14:446-457.

105. Bamias G, Goukos D, Laoudi E, et al. Comparative study of candidate housekeeping genes for quantification of target gene messenger RNA expression by real-time PCR in patients with inflammatory bowel disease. Inflamm Bowel Dis 2013;19: 2840-2847.

106. Sugihara T, Kobori A, Imaeda H, et al. The increased mucosal mRNA expressions of complement C3 and interleukin-17 in inflammatory bowel disease. Clin Exp Immunol 2010;160:386-393.

107. Fransen K, van Sommeren S, Westra HJ, et al. Correlation of genetic risk and messenger RNA expression in a Th17/IL23 pathway analysis in inflammatory bowel disease. Inflamm Bowel Dis 2014;20:777-782.

108. Chiriac MT, Buchen B, Wandersee A, et al. Activation of epithelial signal transducer and activator of transcription 1 by interleukin 28 controls mucosal healing in mice with colitis and is increased in mucosa of patients with inflammatory bowel disease. Gastroenterology 2017;153:123-138.

109. Hong SN, Joung JG, Bae JS, et al. RNA-seq reveals transcriptomic differences in inflamed and noninflamed intestinal mucosa of Crohn's disease patients compared with normal mucosa of healthy controls. Inflamm Bowel Dis 2017;23:1098-1108.

110. Soroosh A, Koutsioumpa M, Pothoulakis C, Iliopoulos D. Functional role and therapeutic targeting of microRNAs in inflammatory bowel disease. Am J Physiol Gastrointest Liver Physiol 2018;314:G256-G262.

111. Tili E, Michaille JJ, Piurowski V, Rigot B, Croce CM. MicroRNAs in intestinal barrier function, inflammatory bowel disease and related cancers-their effects and therapeutic potentials. Curr Opin Pharmacol 2017;37:142-150.

112. Chew CL, Conos SA, Unal B, Tergaonkar V. Noncoding RNAs: Master regulators of inflammatory signaling. Trends $\mathrm{Mol} \mathrm{Med}$ 2018;24:66-84.

113. Yang X, He Q, Guo Z, et al. MicroRNA- 425 facilitates pathogenic Th17 cell differentiation by targeting forkhead box O1 (Foxo1) and is associated with inflammatory bowel disease. Biochem Biophys Res Commun 2018;496:352-358.

114. Rankin CR, Theodorou E, Man Law IK, et al. Identification of novel mRNAs and lncRNAs associated with mouse experimental colitis and human inflammatory bowel disease. Am J Physiol Gastrointest Liver Physiol 2018;315:G722-G733.

115. Castellanos-Rubio A, Kratchmarov R, Sebastian M, et al. Cytoplasmic form of Carlr lncRNA facilitates inflammatory gene expression upon NF- $\kappa B$ Activation. J Immunol 2017;199:581-588.

116. Liao K, Xu J, Yang W, You X, Zhong Q, Wang X. The research progress of LncRNA involved in the regulation of inflammatory diseases. Mol Immunol 2018;101:182-188.

117. Telesco SE, Brodmerkel C, Zhang H, et al. Gene expression signature for prediction of golimumab response in a phase $2 \mathrm{a}$ open-label trial of patients with ulcerative colitis. Gastroenterology 2018;155:1008-1011.

118. Arijs I, Li K, Toedter G, et al. Mucosal gene signatures to predict response to infliximab in patients with ulcerative colitis. Gut 2009; 58:1612-1619.

119. Nunes S, Danesi F, Del Rio D, Silva P. Resveratrol and inflammatory bowel disease: the evidence so far. Nutr Res Rev 2018;31:85-97.

120. Lucafò M, Stankovic B, Kotur N, et al. Pharmacotranscriptomic biomarkers in glucocorticoid treatment of pediatric inflammatory bowel disease. Curr Med Chem 2018;25:2855-2871.

121. Váradi C, Holló Z, Póliska S, et al. Combination of IgG 
$\mathrm{N}$-glycomics and corresponding transcriptomics data to identify anti-TNF- $\alpha$ treatment responders in inflammatory diseases. Electrophoresis 2015;36:1330-1335.

122. Chan PP, Wasinger VC, Leong RW. Current application of proteomics in biomarker discovery for inflammatory bowel disease. World J Gastrointest Pathophysiol 2016;7:27-37.

123. Minic Z, Dahms TES, Babu M. Chromatographic separation strategies for precision mass spectrometry to study proteinprotein interactions and protein phosphorylation. J Chromatogr B Analyt Technol Biomed Life Sci 2018;1102-1103:96-108.

124. Alex P, Gucek M, Li X. Applications of proteomics in the study of inflammatory bowel diseases: Current status and future directions with available technologies. Inflamm Bowel Dis 2009;15:616-629.

125. Meuwis MA, Fillet M, Geurts P, et al. Biomarker discovery for inflammatory bowel disease, using proteomic serum profiling. Biochem Pharmacol 2007;73:1422-1433.

126. Shkoda A, Werner T, Daniel H, Gunckel M, Rogler G, Haller D. Differential protein expression profile in the intestinal epithelium from patients with inflammatory bowel disease. J Proteome Res 2007;6:1114-1125.

127. Nanni P, Mezzanotte L, Roda G, et al. Differential proteomic analysis of HT29 Cl.16E and intestinal epithelial cells by LC ESI/ QTOF mass spectrometry. J Proteomics 2009;72:865-873.

128. Hatsugai M, Kurokawa MS, Kouro T, et al. Protein profiles of peripheral blood mononuclear cells are useful for differential diagnosis of ulcerative colitis and Crohn's disease. J Gastroenterol 2010;45:488-500.

129. Manfredi M, Conte E, Barberis E, et al. Integrated serum proteins and fatty acids analysis for putative biomarker discovery in inflammatory bowel disease. J Proteomics 2019;195:138-149.

130. Drobin K, Assadi G, Hong MG, et al. Targeted analysis of serum proteins encoded at known inflammatory bowel disease risk loci. Inflamm Bowel Dis 2019;25:306-316.

131. Deeke SA, Starr AE, Ning Z, et al. Mucosal-luminal interface proteomics reveals biomarkers of pediatric inflammatory bowel disease-associated colitis. Am J Gastroenterol 2018;113:713-724.

132. Meuwis MA, Fillet M, Lutteri L, et al. Proteomics for prediction and characterization of response to infliximab in Crohn's disease: a pilot study. Clin Biochem 2008;41:960-967.

133. Ferguson LR, Barnett MP. Why are omics technologies important to understanding the role of nutrition in inflammatory bowel diseases? Int J Mol Sci 2016;17.

134. Kang J, Zhu L, Lu J, Zhang X. Application of metabolomics in autoimmune diseases: insight into biomarkers and pathology. J Neuroimmunol 2015;279:25-32.

135. Jansson J, Willing B, Lucio M, et al. Metabolomics reveals metabolic biomarkers of Crohn's disease. PLoS One 2009;4:e6386.

136. Bjerrum JT, Nielsen OH, Hao F, et al. Metabonomics in ulcerative colitis: diagnostics, biomarker identification, and insight into the pathophysiology. J Proteome Res 2010;9:954-962.

137. Diab J, Al-Mahdi R, Gouveia-Figueira S, et al. A quantitative analysis of colonic mucosal oxylipins and endocannabinoids in treatment-naive and deep remission ulcerative colitis patients and the potential link with cytokine gene expression. Inflamm Bowel Dis 2019;25:490-497.

138. Keshteli AH, van den Brand FF, Madsen KL, et al. Dietary and metabolomic determinants of relapse in ulcerative colitis patients: A pilot prospective cohort study. World J Gastroenterol 2017; 23:3890-3899.

139. Scoville EA, Allaman MM, Brown CT, et al. Alterations in lipid, amino acid, and energy metabolism distinguish Crohn's disease from ulcerative colitis and control subjects by serum metabolomic profiling. Metabolomics 2018;14:17.

140. Le Gall G, Noor SO, Ridgway K, et al. Metabolomics of fecal extracts detects altered metabolic activity of gut microbiota in ulcerative colitis and irritable bowel syndrome. J Proteome Res 2011; 10:4208-4218.

141. Dovrolis N, Drygiannakis I, Filidou E, et al. Gut microbial signatures underline complicated Crohn's disease but vary between cohorts; an in silico approach. Inflamm Bowel Dis 2019; 25:217-225

142. Dovrolis N, Kolios G, Spyrou GM, Maroulakou I. Computational profiling of the gut-brain axis: microflora dysbiosis insights to neurological disorders. Brief Bioinform 2017 Nov 27 [Epub ahead of print]. doi: 10.1093/bib/bbx154.

143. Schirmer M, Franzosa EA, Lloyd-Price J, et al. Dynamics of metatranscription in the inflammatory bowel disease gut microbiome. Nat Microbiol 2018;3:337-346.

144. Greenblum S, Turnbaugh PJ, Borenstein E. Metagenomic systems biology of the human gut microbiome reveals topological shifts associated with obesity and inflammatory bowel disease. Proc Natl Acad Sci U S A 2012;109:594-599.

145. Flint HJ, Scott KP, Louis P, Duncan SH. The role of the gut microbiota in nutrition and health. Nat Rev Gastroenterol Hepatol 2012;9:577-589.

146. McDermott AJ, Huffnagle GB. The microbiome and regulation of mucosal immunity. Immunology 2014;142:24-31.

147. Round JL, Mazmanian SK. The gut microbiota shapes intestinal immune responses during health and disease. Nat Rev Immunol 2009;9:313-323.

148. Pickard JM, Zeng MY, Caruso R, Núñez G. Gut microbiota: role in pathogen colonization, immune responses, and inflammatory disease. Immunol Rev 2017;279:70-89.

149. Round JL, Palm NW. Causal effects of the microbiota on immunemediated diseases. Sci Immunol 2018;3.

150. Zeng MY, Inohara N, Nuñez G. Mechanisms of inflammationdriven bacterial dysbiosis in the gut. Mucosal Immunol 2017; 10:18-26.

151. Janssens Y, Nielandt J, Bronselaer A, et al. Disbiome database: linking the microbiome to disease. BMC Microbiol 2018;18:50.

152. Budden KF, Gellatly SL, Wood DL, et al. Emerging pathogenic links between microbiota and the gut-lung axis. Nat Rev Microbiol 2017; 15:55-63.

153. Huffnagle GB, Dickson RP, Lukacs NW. The respiratory tract microbiome and lung inflammation: a two-way street. Mucosal Immunol 2017;10:299-306.

154. Liu R, Hong J, Xu X, et al. Gut microbiome and serum metabolome alterations in obesity and after weight-loss intervention. Nat Med 2017;23:859-868.

155. Bouter KE, van Raalte DH, Groen AK, Nieuwdorp M. Role of the Gut microbiome in the pathogenesis of obesity and obesity-related metabolic dysfunction. Gastroenterology 2017;152:1671-1678.

156. Wu H, Esteve E, Tremaroli V, et al. Metformin alters the gut microbiome of individuals with treatment-naive type 2 diabetes, contributing to the therapeutic effects of the drug. Nat Med 2017;23:850-858.

157. Heintz-Buschart A, May P, Laczny CC, et al. Integrated multiomics of the human gut microbiome in a case study of familial type 1 diabetes. Nat Microbiol 2016;2:16180.

158. Ahmadmehrabi S, Tang WHW. Gut microbiome and its role in cardiovascular diseases. Curr Opin Cardiol 2017;32:761-766.

159. Kasselman LJ, Vernice NA, DeLeon J, Reiss AB. The gut microbiome and elevated cardiovascular risk in obesity and autoimmunity. Atherosclerosis 2018;271:203-213.

160. Jie Z, Xia H, Zhong SL, et al. The gut microbiome in atherosclerotic cardiovascular disease. Nat Commun 2017;8:845.

161. Drosos I, Tavridou A, Kolios G. New aspects on the metabolic role of intestinal microbiota in the development of atherosclerosis. Metabolism 2015;64:476-481.

162. Al Khodor S, Shatat IF. Gut microbiome and kidney disease: a 
bidirectional relationship. Pediatr Nephrol 2017;32:921-931.

163. Nallu A, Sharma S, Ramezani A, Muralidharan J, Raj D. Gut microbiome in chronic kidney disease: challenges and opportunities. Transl Res 2017;179:24-37.

164. Lau WL, Savoj J, Nakata MB, Vaziri ND. Altered microbiome in chronic kidney disease: systemic effects of gut-derived uremic toxins. Clin Sci (Lond) 2018;132:509-522.

165. Routy B, Le Chatelier E, Derosa L, et al. Gut microbiome influences efficacy of PD-1-based immunotherapy against epithelial tumors. Science 2018;359:91-97.

166. Gopalakrishnan V, Helmink BA, Spencer CN, Reuben A, Wargo JA. The influence of the Gut microbiome on cancer, immunity, and cancer immunotherapy. Cancer Cell 2018;33:570-580.

167. Liu K, Lu C. Gut microbes modulate host response to immune checkpoint inhibitor cancer immunotherapy. Transl Cancer Res 2018;7:S608-S610.

168. Flowers SA, Evans SJ, Ward KM, McInnis MG, Ellingrod VL. Interaction between atypical antipsychotics and the Gut microbiome in a bipolar disease cohort. Pharmacotherapy 2017;37:261-267.

169. Vuong HE, Hsiao EY. Emerging roles for the Gut microbiome in autism spectrum disorder. Biol Psychiatry 2017;81:411-423.

170. Cekanaviciute E, Yoo BB, Runia TF, et al. Gut bacteria from multiple sclerosis patients modulate human $\mathrm{T}$ cells and exacerbate symptoms in mouse models. Proc Natl Acad Sci U S A 2017;114:10713-10718.

171. Vogt NM, Kerby RL, Dill-McFarland KA, et al. Gut microbiome alterations in Alzheimer's disease. Sci Rep 2017;7:13537.

172. Sartor RB, Wu GD. Roles for intestinal bacteria, viruses, and fungi in Pathogenesis of inflammatory bowel diseases and therapeutic approaches. Gastroenterology 2017;152:327-339.

173. Kostic AD, Xavier RJ, Gevers D. The microbiome in inflammatory bowel disease: current status and the future ahead. Gastroenterology 2014;146:1489-1499.

174. Wlodarska M, Kostic AD, Xavier RJ. An integrative view of microbiome-host interactions in inflammatory bowel diseases. Cell Host Microbe 2015;17:577-591.

175. Lavelle A, Sokol H. Gut microbiota: beyond metagenomics, metatranscriptomics illuminates microbiome functionality in IBD. Nat Rev Gastroenterol Hepatol 2018;15:193-194.

176. Human Microbiome Project Consortium. Structure, function and diversity of the healthy human microbiome. Nature 2012;486:207-214

177. Cao Y, Shen J, Ran ZH. Association between Faecalibacterium prausnitzii Reduction and inflammatory bowel disease: a metaanalysis and systematic review of the literature. Gastroenterol Res Pract 2014;2014:872725.

178. Lopez-Siles M, Martinez-Medina M, Abellà C, et al. Mucosaassociated Faecalibacterium prausnitzii phylotype richness is reduced in patients with inflammatory bowel disease. Appl Environ Microbiol 2015;81:7582-7592.

179. Hedin CR, van der Gast CJ, Stagg AJ, Lindsay JO, Whelan K. The gut microbiota of siblings offers insights into microbial pathogenesis of inflammatory bowel disease. Gut Microbes 2017; 8:359-365.

180. Robinson AM, Gondalia SV, Karpe AV, et al. Fecal microbiota and metabolome in a mouse model of spontaneous chronic colitis: relevance to human inflammatory bowel disease. Inflamm Bowel Dis 2016;22:2767-2787.

181. Chu H, Khosravi A, Kusumawardhani IP, et al. Gene-microbiota interactions contribute to the pathogenesis of inflammatory bowel disease. Science 2016;352:1116-1120.

182. Halfvarson J, Brislawn CJ, Lamendella R, et al. Dynamics of the human gut microbiome in inflammatory bowel disease. Nat Microbiol 2017;2:17004.

183. Dovrolis N, Drygiannakis I, Filidou E, et al. Gut microbial signatures underline complicated Crohn's disease but vary between cohorts; an in silico approach. Inflamm Bowel Dis 2018; 25:217-225.

184. Ananthakrishnan AN, Luo C, Yajnik V, et al. Gut microbiome function predicts response to anti-integrin biologic therapy in inflammatory bowel diseases. Cell Host Microbe 2017;21:603-610.

185. Chin SM, Sauk J, Mahabamunuge J, Kaplan JL, Hohmann EL, Khalili H. Fecal microbiota transplantation for recurrent Clostridium difficile infection in patients with inflammatory bowel disease: a single-center experience. Clin Gastroenterol Hepatol 2017;15:597-599.

186. Anderson JL, Edney RJ, Whelan K. Systematic review: faecal microbiota transplantation in the management of inflammatory bowel disease. Aliment Pharmacol Ther 2012;36:503-516.

187. Colman RJ, Rubin DT. Fecal microbiota transplantation as therapy for inflammatory bowel disease: a systematic review and meta-analysis. J Crohns Colitis 2014;8:1569-1581.

188. Goyal A, Yeh A, Bush BR, et al. Safety, clinical response, and microbiome findings following fecal microbiota transplant in children with inflammatory bowel disease. Inflamm Bowel Dis 2018;24:410-421.

189. Newman KM, Rank KM, Vaughn BP, Khoruts A. Treatment of recurrent Clostridium difficile infection using fecal microbiota transplantation in patients with inflammatory bowel disease. Gut Microbes 2017;8:303-309.

190. Qazi T, Amaratunga T, Barnes EL, Fischer M, Kassam Z, Allegretti JR. The risk of inflammatory bowel disease flares after fecal microbiota transplantation: Systematic review and metaanalysis. Gut Microbes 2017;8:574-588.

191. Fischer M, Bittar M, Papa E, Kassam Z, Smith M. Can you cause inflammatory bowel disease with fecal transplantation? A 31-patient case-series of fecal transplantation using stool from a donor who later developed Crohn's disease. Gut Microbes 2017; 8:205-207.

192. Aliper A, Plis S, Artemov A, Ulloa A, Mamoshina P, Zhavoronkov A. Deep learning applications for predicting pharmacological properties of drugs and drug repurposing using transcriptomic data. Mol Pharm 2016;13:2524-2530.

193. Berenstein AJ, Magariños MP, Chernomoretz A, Agüero F. A multilayer network approach for guiding drug repositioning in neglected diseases. PLoS Negl Trop Dis 2016;10:e0004300.

194. Mullen J, Cockell SJ, Woollard P, Wipat A. An integrated data driven approach to drug repositioning using gene-disease associations. PLoS One 2016;11:e0155811.

195. Singh P, Dhaneshwar SS. Investigating drug repositioning approach to design novel prodrugs for colon-specific release of fexofenadine for ulcerative colitis. Curr Drug Deliv 2017;14: 543-554.

196. Dudley JT, Sirota M, Shenoy M, et al. Computational repositioning of the anticonvulsant topiramate for inflammatory bowel disease. Sci Transl Med 2011;3:96ra76.

197. Clark PM, Dawany N, Dampier W, Byers SW, Pestell RG, Tozeren A. Bioinformatics analysis reveals transcriptome and microRNA signatures and drug repositioning targets for IBD and other autoimmune diseases. Inflamm Bowel Dis 2012;18: 2315-2333.

198. Cai X, Chen Y, Gao Z, Xu R. Explore small molecule-induced genome-wide transcriptional profiles for novel inflammatory bowel disease Drug. AMIA Jt Summits Transl Sci Proc 2016; 2016:22-31.

199. Collij V, Festen EA, Alberts R, Weersma RK. Drug repositioning in inflammatory bowel disease based on genetic information. Inflamm Bowel Dis 2016;22:2562-2570.

200. Paramsothy S, Rosenstein AK, Mehandru S, Colombel JF. The current state of the art for biological therapies and new small 
molecules in inflammatory bowel disease. Mucosal Immunol 2018; 11:1558-1570.

201. Weisshof R, El Jurdi K, Zmeter N, Rubin DT. Emerging therapies for inflammatory bowel disease. Adv Ther 2018;35:1746-1762.

202. D’Amico F, Fiorino G, Furfaro F, Allocca M, Danese S. Janus kinase inhibitors for the treatment of inflammatory bowel diseases: developments from phase I and phase II clinical trials. Expert Opin Investig Drugs 2018;27:595-599.

203. Allen S, Moran N. Cell Adhesion Molecules: Therapeutic targets for inhibition of inflammatory states. Semin Thromb Hemost 2015; 41:563-571.

204. Holleran G, Lopetuso L, Petito V, et al. The innate and adaptive immune system as targets for biologic therapies in inflammatory bowel disease. Int J Mol Sci 2017;18.

205. Nesvizhskii AI. Proteogenomics: concepts, applications and computational strategies. Nat Methods 2014;11:1114-1125.

206. Menschaert G, Fenyö D. Proteogenomics from a bioinformatics angle: A growing field. Mass Spectrom Rev 2017;36:584-599.

207. Roumpeka DD, Wallace RJ, Escalettes F, Fotheringham I, Watson M. A Review of bioinformatics tools for bio-prospecting from metagenomic sequence data. Front Genet 2017;8:23.

208. Backofen R, Engelhardt J, Erxleben A, et al. RNA-bioinformatics: tools, services and databases for the analysis of RNA-based regulation. J Biotechnol 2017;261:76-84.

209. Breitwieser FP, Lu J, Salzberg SL. A review of methods and databases for metagenomic classification and assembly. Brief Bioinform 2017 Sep 23 [Epub ahead of print]. doi: 10.1093/bib/ bbxi20.

210. Zhang Z, Li H, Jiang S, et al. A survey and evaluation of Webbased tools/databases for variant analysis of TCGA data. Brief Bioinform 2018 Mar 29 [Epub ahead of print]. doi: 10.1093/bib/bby023.

211. Nithin C, Ghosh P, Bujnicki J. Bioinformatics tools and benchmarks for computational docking and 3D structure prediction of RNA-protein complexes. Genes (Basel) 2018;9.

212. de Souza HSP, Fiocchi C, Iliopoulos D. The IBD interactome: an integrated view of aetiology, pathogenesis and therapy. Nat Rev Gastroenterol Hepatol 2017; 14:739-749.

213. Alon U. Biological networks: the tinkerer as an engineer. Science 2003;301:1866-1867.

214. Warde-Farley D, Donaldson SL, Comes O, et al. The GeneMANIA prediction server: biological network integration for gene prioritization and predicting gene function. Nucleic Acids Res 2010;38:W214-W220.

215. Szklarczyk D, Morris JH, Cook H, et al. The STRING database in 2017: quality-controlled protein-protein association networks, made broadly accessible. Nucleic Acids Res 2017;45:D362-D368.

216. Kanehisa M, Araki M, Goto S, et al. KEGG for linking genomes to life and the environment. Nucleic Acids Res 2008;36:D480-D484.

217. Shannon P, Markiel A, Ozier O, et al. Cytoscape: a software environment for integrated models of biomolecular interaction networks. Genome Res 2003;13:2498-2504.

218. Csardi G, Nepusz T. The igraph software package for complex network research. InterJournal, Complex Systems 2006;1695:1-9.

219. Peters LA, Perrigoue J, Mortha A, et al. A functional genomics predictive network model identifies regulators of inflammatory bowel disease. Nat Genet 2017;49:1437-1449.

220. Benchimol EI, Bernstein CN, Bitton A, et al. Trends in epidemiology of pediatric inflammatory bowel disease in Canada: distributed network analysis of multiple populationbased provincial health administrative databases. Am J Gastroenterol 2017;112:1120-1134.

221. Coward S, Kuenzig ME, Hazlewood G, et al. Comparative effectiveness of mesalamine, sulfasalazine, corticosteroids, and budesonide for the induction of remission in Crohn's disease: a bayesian network meta-analysis: republished. Inflamm Bowel Dis 2017;23:E26-E37. 